\title{
Cannonballs in the context of gamma ray bursts ${ }^{\star}$
}

\section{Formation sites?}

\author{
J. E. Staff ${ }^{1}$, C. Fendt ${ }^{2}$, and R. Ouyed ${ }^{1,3}$ \\ ${ }^{1}$ Department of Physics and Astronomy, University of Calgary, 2500 University Drive NW, Calgary, Alberta, T2N 1N4, Canada \\ e-mail: jstaff@capca.ucalgary.ca \\ 2 Max-Planck-Institut für Astronomie, Königstuhl 17, 69117 Heidelberg, Germany \\ 3 Canadian Institute for Theoretical Astrophysics, 60 St. George Street, Toronto, Ontario, Canada
}

Received 12 October 2005 / Accepted 1 December 2005

\section{ABSTRACT}

We investigate possible formation sites of the cannonballs (in the gamma ray bursts context) by calculating their physical parameters, such as density, magnetic field, and temperature close to the origin. Our results favor scenarios where the cannonballs form as instabilities (knots) within magnetized jets from hyperaccreting disks. These instabilities would most likely set in beyond the light cylinder where flow velocity with Lorentz factors as high as 2000 can be achieved. The cannonball model for gamma ray bursts requires that cannonballs form inside corecollapse supernovae. Our findings challenge the cannonball model of gamma ray bursts, unless hyperaccreting disks and the corresponding jets are common occurrences in core-collapse SNe.

Key words. gamma rays: bursts

\section{Introduction}

It has been argued in the literature that, as an alternative to the fireball scenario (e.g. Piran 1999, and references therein), the so-called cannonball (CB) model provides a good fit to the observed GRB flux and temporal variations (Dar \& De Rújula 2004). For example, to explain GRBs, CBs must be created in supernova explosions and accelerated to high Lorentz factors, $\Gamma_{\mathrm{CB}} \sim 1000$. However, the origin of these highly relativistic "balls" of matter has not yet been investigated and is the subject of much debate. In order to shed some light on the still open questions of their formation and early evolution, this paper investigates the physical conditions at the origin at which given their features at the distance of CBs become transparent to their enclosed radiation as required to explain GRBs. Our proposal is that the conditions within the $\mathrm{CB}$ as we scale the distance down along its path to the origin should be an indication of their formation site. Despite the simplicity of our approach, we hope this study will help elucidate some questions related to the origin/existence of these CBs. We start in Sect. 2 by a brief introduction to the $\mathrm{CB}$ model as described in Dar \& De Rújula (2004). In particular we isolate the conditions of CBs at large distances from the source that best fit GRB light curves. In Sect. 3 we present the methods we adopted to extrapolate back to the CB source. In Sect. 4, we study

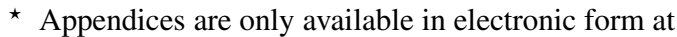
http://www. edpsciences.org possible formation sites and explore formation mechanisms given the conditions at the origin. Section 5 is devoted to the study of mechanisms capable of accelerating CBs to Lorentz factors as high as $\sim 1000$. We summarize our results and conclude in Sect. 6.

\section{The CB model for GRBs}

In the $\mathrm{CB}$ model for GRBs, the prompt gamma ray emission is assumed to be produced when ambient light from the supernova $^{1}$ is Compton up-scattered by the electrons in the CB. These CBs move with $\Gamma_{\mathrm{CB}} \sim 1000$ with respect to the supernova remnant, and as such, the emitted radiation is highly beamed in the observer frame (Dar \& De Rújula 2004). For the first $10^{3} \mathrm{~s}$ in the $\mathrm{CB}$ rest frame, the $\mathrm{CB}$ in a fast-cooling phase emits via thermal bremsstrahlung (Dado et al. 2002), but eventually, its emissivity is dominated by synchrotron emission from ISM electrons that penetrate it, it is argued. A CB will become transparent to the bulk of its enclosed radiation in a time of $O(1) \mathrm{s}$ in the observer frame, after it exits the transparent outskirts of the shell of the associated SN. The internal radiation pressure drops abruptly, and its transverse expansion rate is quenched by collisionless, magnetic-field mediated interactions with the ISM (Dado et al. 2002).

\footnotetext{
1 The wind from the SN progenitor star is ionized and is semitransparent to photons in the visible and UV frequencies.
} 
Table 1. Cannonball parameters as given by Dado et al. (2002) and Dar \& De Rújula (2004).

\begin{tabular}{ll}
\hline \hline Parameter & Value \\
\hline$R_{\mathrm{CB}, \max }$ & $2.2 \times 10^{14} \mathrm{~cm}$ \\
$R_{\mathrm{CB}, \text { trans }}$ & $10^{13} \mathrm{~cm}$ \\
$D_{\text {trans }}$ & $1.7 \times 10^{16} \mathrm{~cm}$ \\
$\Gamma_{\mathrm{CB}}$ & $1.0 \times 10^{3}$ \\
$\delta$ & $1.0 \times 10^{3}$ \\
$z$ & 1 \\
$\beta_{\mathrm{i}}$ & $1 / \sqrt{3}$ \\
$N_{\mathrm{CB}}$ & $10^{50}$ \\
$M_{\mathrm{CB}}$ & $10^{26} \mathrm{~g}$ \\
\hline
\end{tabular}

Typical values for CB parameters as derived by Dado et al. (2002) and Dar \& De Rújula (2004) are given in Table 1 where we denote the radius of the $\mathrm{CB}$ by $R_{\mathrm{CB}}$, the distance travelled by the CB from its origin by $D$, the time passed in the CB rest frame by $t$. The expansion velocity of the CB is denoted by $\beta_{i}=$ $v_{i} / c$, the number of baryons, and the mass of the CB by $N_{\mathrm{CB}}$ and $M_{\mathrm{CB}}$, respectively. The subscript "trans" refers to the point where the CB becomes transparent ("transparency radius").

By fitting the observed GRB afterglow, the CB Lorentz factor is estimated to vary between $\Gamma_{\mathrm{CB}}=250$ and $\Gamma_{\mathrm{CB}}=1600$, while the number of baryons is on the order of $10^{50}$. With this information at hand, our goal is to derive the conditions in the $\mathrm{CB}$ as we integrate back to a plausible source. As an indication of the close proximity to a compact source (e.g. black-holes and neutron stars), when applicable we will make use of the notion of light cylinder, which we take to be about $R_{\mathrm{L}} \sim 10^{7} \mathrm{~cm}$.

\section{Cannonball propagation and evolution}

In this section, we explore the evolution of the CB by extrapolating backward from the location where the GRB occurs to where the $\mathrm{CB}$ reaches nuclear saturation density (applying CB parameters as given in Table 1). For simplicity, we assume that the $\mathrm{CB}$ is expanding with a constant velocity and is moving with a constant Lorentz factor. The natural assumption for the expansion velocity is the sound speed of the hot blob of matter, $v_{\text {exp }} \simeq c_{\mathrm{s}} \simeq c / \sqrt{3}$. Six different cases of CB Lorentz factor and mass are investigated (see Table 2).

We first apply a simple model of the CB's internal energy obeying a simple equation of state. Using this we calculate the evolution of the density, magnetic field, and temperature as the $\mathrm{CB}$ moves away from the origin. In a second step we extend our model approach by applying an energy equation where pressure degeneracy and neutrino effects are included. As we will see, spatial back integration from the transparency radius strongly indicates that the $\mathrm{CB}$ may be launched close to a black hole. The spatial integration back to the source is carried out until the CB temperature reaches extreme values, $T_{1} \sim 100 \mathrm{MeV}$, unless the $\mathrm{CB}$ density reaches nuclear saturation density before $T_{1}$.

With the CB expanding at a constant expansion velocity equal to the sound speed of the matter $v_{\exp }=c / \sqrt{3}$, we can use
Table 2. The different cases of CBs explored in this work. Note that cases 6,8 , and $9\left(\Gamma_{\mathrm{CB}}=1000\right.$ and $N_{\mathrm{CB}}=10^{51}, \Gamma_{\mathrm{CB}}=2000$ and $N_{\mathrm{CB}}=10^{50}, \Gamma_{\mathrm{CB}}=2000$ and $N_{\mathrm{CB}}=10^{51}$, respectively) are not consistent with our assumptions as they reach nuclear saturation density at a distance beyond the light cylinder, so these were left out in this paper.

\begin{tabular}{lll}
\hline \hline & $\Gamma_{\mathrm{CB}}$ & $N_{\mathrm{CB}}$ \\
\hline Case 1 & $1.0 \times 10^{2}$ & $10^{49}$ \\
Case 2 & $1.0 \times 10^{2}$ & $10^{50}$ \\
Case 3 & $1.0 \times 10^{2}$ & $10^{51}$ \\
\hline Case 4 & $1.0 \times 10^{3}$ & $10^{49}$ \\
Case 5 & $1.0 \times 10^{3}$ & $10^{50}$ \\
\hline Case 7 & $2.0 \times 10^{3}$ & $10^{49}$ \\
\hline
\end{tabular}

Table 3. The CB radius at the point where it reaches nuclear saturation density (left), at the light cylinder $R_{\mathrm{LC}}=1.5 \times 10^{7} \mathrm{~cm}$ (middle), and at the distance where the $\mathrm{CB}$ becomes transparent to radiation (right).

\begin{tabular}{llll}
\hline \hline & $R_{\mathrm{CB}, \mathrm{nuc}}[\mathrm{cm}]$ & $R_{\mathrm{CB}, \mathrm{LC}}[\mathrm{cm}]$ & $R_{\mathrm{CB}, \text { trans }}[\mathrm{cm}]$ \\
\hline Case 1 & $2.5 \times 10^{3}$ & $1.2 \times 10^{5}$ & $1.0 \times 10^{12}$ \\
Case 2 & $5.6 \times 10^{3}$ & $1.2 \times 10^{5}$ & $1.0 \times 10^{13}$ \\
Case 3 & $12.0 \times 10^{3}$ & $1.2 \times 10^{5}$ & $1.0 \times 10^{14}$ \\
\hline Case 4 & $2.5 \times 10^{3}$ & $1.2 \times 10^{4}$ & $1.0 \times 10^{12}$ \\
Case 5 & $5.6 \times 10^{3}$ & $1.2 \times 10^{4}$ & $1.0 \times 10^{13}$ \\
\hline Case 7 & $2.5 \times 10^{3}$ & $5.8 \times 10^{3}$ & $1.0 \times 10^{12}$ \\
\hline
\end{tabular}

certain estimates about the $\mathrm{CB}$ at the distance of transparency to derive an interrelation between radius and distance the $\mathrm{CB}$ has traveled from its origin. As the radius of the CB at the distance of transparency, we apply the estimate by Dado et al. (2002),

$R_{\text {trans }} \simeq 10^{13}\left(\frac{N_{\mathrm{CB}}}{6 \times 10^{50}}\right)^{1 / 2} \mathrm{~cm}$.

To reach this radius, the $\mathrm{CB}$ has traveled a period of time

$t_{\text {trans, } \mathrm{CB}}=\frac{R_{\text {trans }}}{v_{\text {exp }}} \simeq 577\left(\frac{N_{\mathrm{CB}}}{10^{50}}\right)^{1 / 2} \mathrm{~s}$

in the $\mathrm{CB}$ rest frame. As it travels essentially with the speed of light, at the time when it becomes transparent, the $\mathrm{CB}$ has traveled a distance

$D_{\text {trans }}=\Gamma_{\mathrm{CB}} c t_{\text {trans }}$

from its origin where it was ejected. This gives a linear scaling factor

$l=R_{\text {trans }} / D_{\text {trans }}=\frac{1}{\Gamma_{\mathrm{CB}} \sqrt{3}} \cdot$

The radius of a $\mathrm{CB}$ is then simply expressed as $R_{\mathrm{CB}}=$ $D / \Gamma_{\mathrm{CB}} \sqrt{3}$. The radius at nuclear saturation density and at the light cylinder $\left(D_{\mathrm{lc}} \sim 1.5 \times 10^{7} \mathrm{~cm}\right)$ are listed in Table 3 , along with the radius at which the $\mathrm{CB}$ becomes transparent to the enclosed radiation for the different cases of $\mathrm{CB}$ masses and Lorentz factors. 


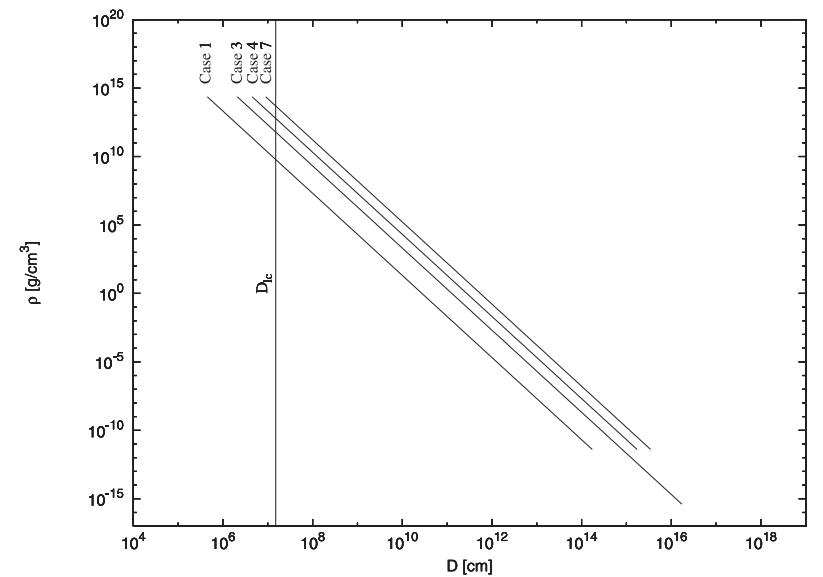

Fig. 1. Density vs. distance from the origin for the CB. The backward integration is stopped when the $\mathrm{CB}$ density reaches nuclear saturation density.

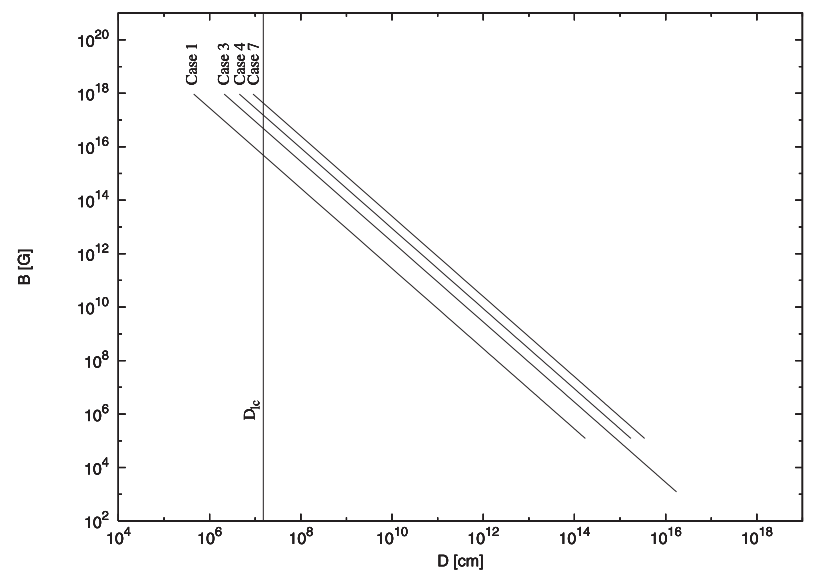

Fig. 2. Magnetic field vs. distance from the origin for the CB. The backward integration is stopped when the $\mathrm{CB}$ density reaches nuclear saturation density.

Figure 1 shows density as a function of distance travelled by the $\mathrm{CB}$. Depending on the $\mathrm{CB}$ parameters, the densities at the light cylinder range from $10^{10}-10^{14} \mathrm{~g} / \mathrm{cm}^{3}$. If we estimate the CB magnetic field strength from the equipartition assumption, $v_{\mathrm{A}} \simeq v_{\mathrm{s}}=c / \sqrt{3}$, with the Alfvén speed $v_{\mathrm{A}}$ and

$B=v_{\mathrm{A}} \sqrt{4 \pi \rho}=c \sqrt{\frac{M_{\mathrm{CB}}}{R_{\mathrm{CB}}^{3}}}$,

we may compute the $\mathrm{CB}$ magnetic field versus the distance from origin (Fig. 2). Close to the hypothetical CB origin where we reach nuclear saturation density, the magnetic field strength approaches values up to $B \sim 10^{18} \mathrm{G}$ (see also Sect. 4.1). At a distance from the origin on the order of the light cylinder radius, the field strength is between $4.8 \times 10^{15}$ and $1.4 \times 10^{18}$ Gauss depending on the choice of Lorentz factor and mass of the CB. The density ranges from $10^{9}$ to $10^{14} \mathrm{~g} / \mathrm{cm}^{3}$.

\subsection{Simple energy equation}

We continue our simple estimates by assuming energy conservation in the $C B$

$E_{\mathrm{rad}}+E_{\mathrm{th}}+E_{\mathrm{mag}}=E_{\mathrm{tot}}$.

The radiation energy is written as

$E_{\mathrm{rad}}=a T^{4} \frac{4}{3} \pi R_{\mathrm{CB}}^{3}$,

the magnetic energy is

$E_{\mathrm{mag}}=\epsilon_{\mathrm{m}} M_{\mathrm{CB}} c^{2}$,

and the gas thermal energy is

$E_{\mathrm{th}}=3 N_{\mathrm{CB}} k T$,

where $a=7.5657 \times 10^{-15} \mathrm{erg} \mathrm{cm}^{-3} \mathrm{~K}^{-4}$ is the radiation constant, $k=1.3807 \times 10^{-16} \mathrm{erg} \mathrm{K}^{-1}$ is Boltzmann's constant, $T$ the $\mathrm{CB}$ temperature, $\epsilon_{\mathrm{m}}$ a parameter that allows us to write the magnetic energy in terms of the $\mathrm{CB}$ rest mass energy. This parameter is fixed by imposing energy equipartition at the specified CB origin. Note that the magnetic energy is constant, as we assume that the magnetic field is not dissipated in reconnection events and is not expelled from the CB. The gravitational energy is always negligible compared to the other energy channels. The total energy is then given as:

$E_{\mathrm{tot}}=3 \times E_{\mathrm{mag}}=3 \epsilon_{\mathrm{m}} M_{\mathrm{CB}} c^{2}$.

We can now write the energy equation as:

$4 / 3 \pi R^{3} a T^{4}+3 N k T=2 \epsilon_{\mathrm{m}} M c^{2}$.

This equation is solved to find $T$ as a function of $D$.

In what follows, we explore two scenarios: (i) the first one is indicative of the close proximity of a compact source, and as such it corresponds to the case where the energy equation is integrated assuming equipartition at nuclear saturation densities; (ii) the second reflects scenarios where the $\mathrm{CB}$ originates in the coronal region of compact stars of their associated accretion disks; see the specifics below.

\subsubsection{Equipartition at nuclear densities: source origin}

To set equipartition at nuclear saturation density, $\epsilon_{\mathrm{m}}$ has to be 0.5 for all cases. By rearranging Eq. (11) it can be seen that it becomes a function of $T$ and $\rho$ :

$a T^{4}+3 \rho k T / m_{\mathrm{H}}=2 \rho c^{2} \epsilon_{\mathrm{m}}$.

The temperature therefore depends only on the density and implies a temperature of about $10^{12} \mathrm{~K}$ at nuclear saturation density for all cases. The temperature as a function of distance travelled is shown in Fig. 3, while Fig. 4 shows the energy components for case 4 . The radiation energy is dominant everywhere except at nuclear saturation density where there is equipartition. We note that nuclear saturation density is reached at distances larger than $10^{6} \mathrm{~cm}$ for most cases. It is unrealistic to find objects with such high densities that are much larger than $10^{6} \mathrm{~cm}$. Also, $\mathrm{CBs}$ with such densities need a magnetic field $B=10^{18} \mathrm{G}$, 


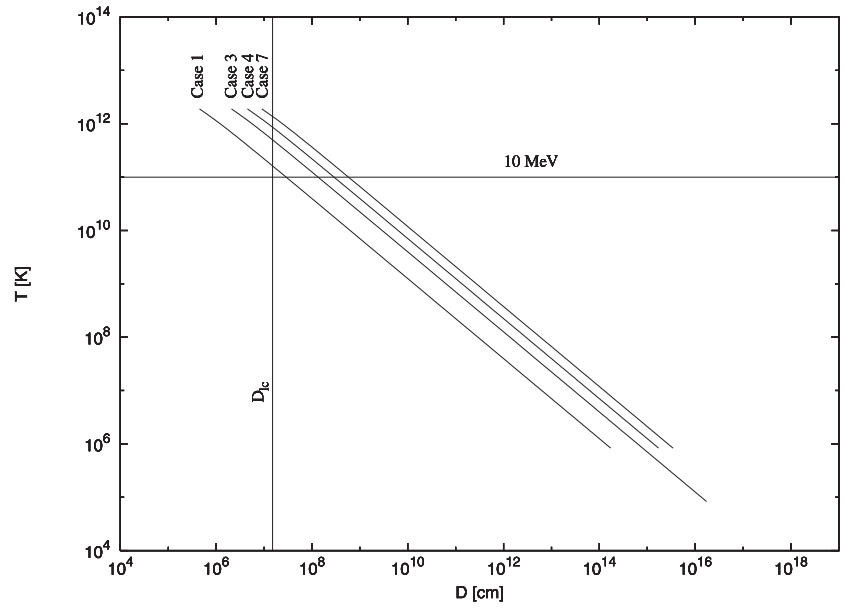

Fig. 3. Temperature vs. distance traveled by the $\mathrm{CB}$ assuming equipartition at nuclear saturation density.

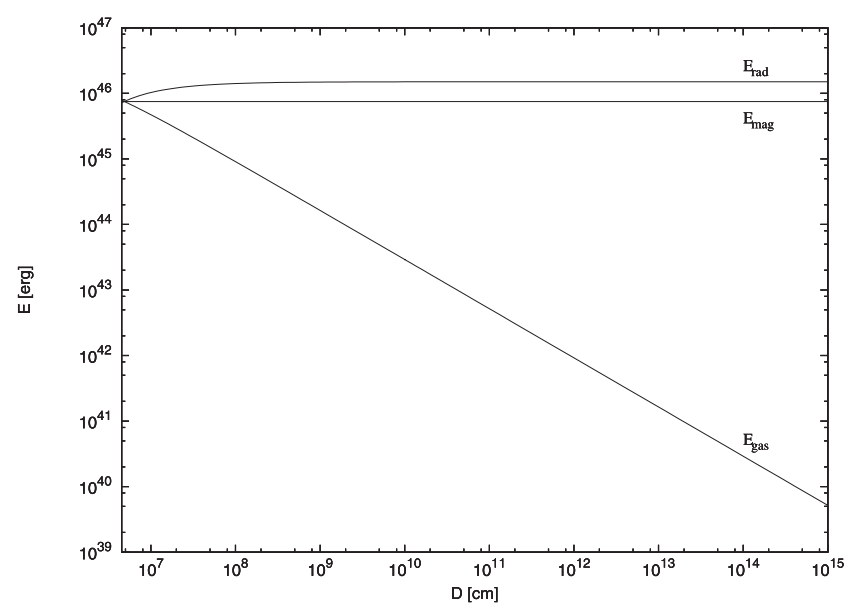

Fig. 4. The different energy components for case 4 using the simple energy equation and assuming equipartition at nuclear densities.

which is unrealistically high. We can therefore rule out CBs formed with nuclear saturation density.

It should be noted that the solutions are not very sensitive to the choice of total internal energy ${ }^{2}$. As an example, increasing the total internal energy by an order of magnitude, we find a temperature at the light cylinder, $D_{\text {lc }}$, for case 3 to be $9 \times 10^{11} \mathrm{~K}$, compared to $5 \times 10^{11} \mathrm{~K}$ in our initial calculation.

\subsubsection{Equipartition at the light cylinder: coronal origin}

Figure 5 shows the evolution of the $\mathrm{CB}$ conditions when equipartition at a distance of about a light cylinder radius is assumed, and Fig. 6 shows the energy components for case 4 . Table 4 shows the temperature found at the light cylinder and the value for the energy equipartition parameter $\epsilon_{\mathrm{m}}$ for different kinematic parameters of the CB (see Table1). Note that $\epsilon_{\mathrm{m}}$ is now determined by the condition that we have equipartition at the $\mathrm{CB}$ origin (i.e. at a light cylinder distance).

\footnotetext{
${ }^{2}$ The expansion energy of the $\mathrm{CB}$ is the same order as the magnetic energy. For simplicity we have included it in the expression for the total energy.
}

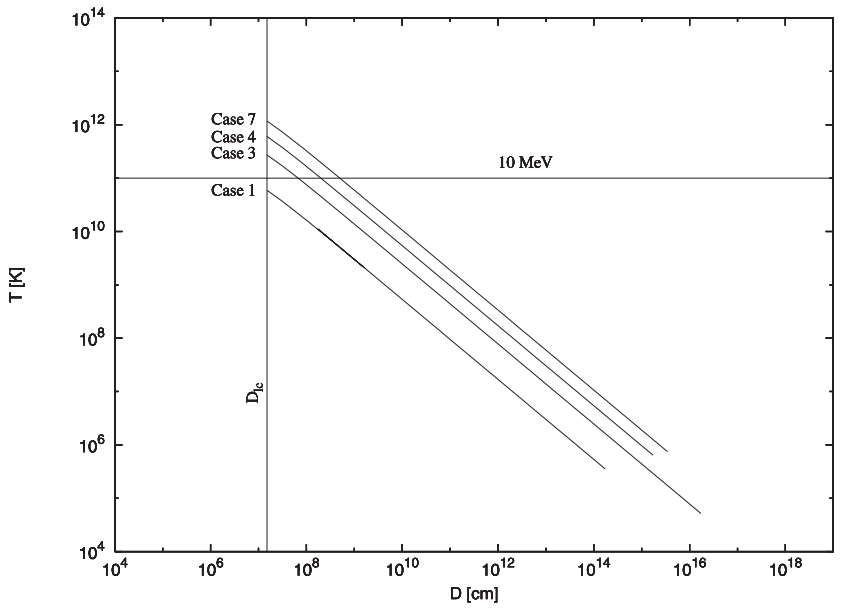

Fig. 5. Temperature vs. distance traveled by the CB assuming equipartition at the light cylinder.

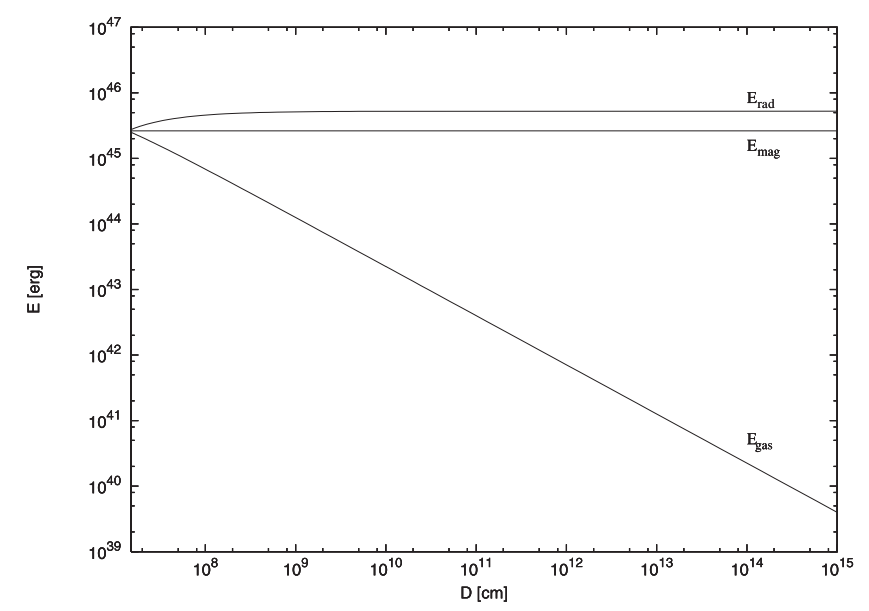

Fig. 6. The different energy components for case 4 using the simple energy equation and assuming equipartition at the light cylinder.

Table 4. Coronal $\mathrm{CB}$ origin, showing the values for the temperature and $\epsilon_{\mathrm{m}}$ found for the simple energy equation assuming equipartition between the magnetic, gas thermal, and radiation energy at the light cylinder.

\begin{tabular}{lll}
\hline \hline Case & $T[\mathrm{~K}]$ & $\epsilon_{\mathrm{m}}$ \\
\hline 1 & $5.9 \times 10^{10}$ & 0.0165 \\
2 & $1.3 \times 10^{11}$ & 0.035 \\
3 & $2.7 \times 10^{11}$ & 0.075 \\
4 & $6.1 \times 10^{11}$ & 0.175 \\
5 & $1.2 \times 10^{12}$ & 0.325 \\
7 & $1.2 \times 10^{12}$ & 0.325 \\
\hline
\end{tabular}

By assuming an equal number of electrons $n_{\mathrm{e}}$ and baryons in the $\mathrm{CB}$, the Fermi temperature can be computed. If we take the light cylinder distance as a typical length unit at the $\mathrm{CB}$ origin and compute the Fermi temperature at this location, we see that the $\mathrm{CB}$ temperature is much lower than the electron Fermi temperature (Table 5). We therefore have to improve our approach to also consider electron degeneracy and neutrino effects. In the next section we explore a more appropriate 
Table 5. The values for the magnetic field, temperature and density found at the light cylinder for the simple energy equation. The temperature and magnetic field at nuclear saturation density is $1.2 \times 10^{12} \mathrm{~K}$ and $9.3 \times 10^{17} \mathrm{G}$, respectively, for all cases, as both the magnetic field strength and temperature are only dependent on the density.

\begin{tabular}{lllll}
\hline \hline Case & $B[\mathrm{G}]$ & $T[\mathrm{~K}]$ & $T_{\mathrm{F}}[\mathrm{K}]$ & $\rho\left[\mathrm{g} \mathrm{cm}^{-3}\right]$ \\
\hline 1 & $4.8 \times 10^{15}$ & $5.9 \times 10^{10}$ & $5.6 \times 10^{14}$ & $6.1 \times 10^{9}$ \\
2 & $1.5 \times 10^{16}$ & $1.3 \times 10^{11}$ & $2.6 \times 10^{15}$ & $6.1 \times 10^{10}$ \\
3 & $4.8 \times 10^{16}$ & $2.7 \times 10^{11}$ & $1.2 \times 10^{16}$ & $6.1 \times 10^{11}$ \\
4 & $1.5 \times 10^{17}$ & $6.0 \times 10^{11}$ & $5.6 \times 10^{16}$ & $6.1 \times 10^{12}$ \\
5 & $4.8 \times 10^{17}$ & $1.2 \times 10^{12}$ & $2.6 \times 10^{17}$ & $6.1 \times 10^{13}$ \\
7 & $4.3 \times 10^{17}$ & $1.2 \times 10^{11}$ & $2.3 \times 10^{17}$ & $4.9 \times 10^{13}$ \\
\hline
\end{tabular}

energy equation where neutrino effects are added. However, we still apply a kinematic approach assuming a linear expansion of the $\mathrm{CB}$.

\subsection{Coronal $C B$ origin: neutrino effects}

In this section we improve our approach by taking degeneracy pressure and neutrino cooling (e.g. Popham et al. 1999) into account. The energy equation becomes

$E_{\mathrm{tot}}+E_{v}(t)=E_{\mathrm{th}}+E_{\mathrm{rad}}+E_{\mathrm{deg}}+E_{\mathrm{mag}}$

where

$E_{\mathrm{deg}}=3 K M_{\mathrm{CB}}\left(\frac{\rho}{\mu_{\mathrm{e}}}\right)^{1 / 3}$

is the degeneracy energy,

$E_{\mathrm{rad}}=\frac{11}{4} \frac{a T^{4}}{\rho} M_{\mathrm{CB}}$

is the radiation energy, and

$E_{\mathrm{th}}=\frac{3}{2} R T M_{\mathrm{CB}} \frac{1+3 X_{\mathrm{nuc}}}{4}$

the gas thermal energy, where

$$
\begin{aligned}
X_{\text {nuc }}= & 30.97\left(\frac{\rho}{10^{10} \mathrm{~g} / \mathrm{cm}^{3}}\right)^{-3 / 4}\left(\frac{T}{10^{10} \mathrm{~K}}\right)^{9 / 8} \\
& \times \exp \left(-6.096 \times \frac{10^{10} \mathrm{~K}}{T}\right)
\end{aligned}
$$

gives $X_{\text {nuc }}<1$ and $X_{\text {nuc }}=1$ elsewhere. In the equation above, $K=(2 \pi h c / 3)\left(3 / 8 \pi m_{\mathrm{n}}\right)^{4 / 3}=1.24 \times 10^{15}, m_{\mathrm{n}}$ is the nucleon mass, $R$ the gas constant, $a$ the radiation constant and $\mu_{\mathrm{e}}=2$ the mass per electron. Inserting Eqs. (8), (14), (15) and (16) into (13) gives, in terms of $D$ :

$$
\begin{aligned}
E_{\mathrm{tot}}+E_{v}(t)= & 3 K M_{\mathrm{CB}}\left(\frac{M_{\mathrm{CB}} 3^{5 / 2}}{4 \pi \mu_{\mathrm{e}}}\right)^{1 / 3} \frac{\Gamma_{\mathrm{CB}}}{D} \\
& +\frac{3}{2} R T M_{\mathrm{CB}} \frac{1+3 X_{\mathrm{nuc}}}{4}+\frac{11}{4} a T^{4} \frac{4 \pi D^{3}}{\Gamma_{\mathrm{CB}}^{3} 3^{5 / 2}} .
\end{aligned}
$$

Note that we apply the same total internal energy $\left(E_{\text {tot }}\right)$ of the $\mathrm{CB}$ as in Sect. 3.3, thus the same energy parameter $\epsilon_{\mathrm{m}}$. Close to the origin, however, we add an energy component due to neutrino effects (emissivity and cooling) and is denoted by $E_{v}(t)$ in Eq. (13).

Two types of neutrino losses may occur, i.e. neutrino emission due to pair annihilation and neutrino losses due to the capture of pairs on nuclei. Their contribution to the energy budget is computed from Eqs. (3.8) and (3.9) in Popham et al. (1999):

$$
\begin{aligned}
& \dot{q}_{v \bar{v}}=5.0 \times 10^{33}\left(\frac{T}{10^{11} \mathrm{~K}}\right)^{9} \operatorname{erg~cm}^{-3} \mathrm{~s}^{-1} . \\
& \dot{q}_{\mathrm{e} N}=9.0 \times 10^{33}\left(\frac{\rho}{10^{10} \mathrm{~g} / \mathrm{cm}^{3}}\right)\left(\frac{T}{10^{11} \mathrm{~K}}\right)^{6} \operatorname{erg~cm}^{-3} \mathrm{~s}^{-1} .
\end{aligned}
$$

These expressions are integrated over the time it takes the $\mathrm{CB}$ to reach conditions for which neutrino cooling is not significant. We find the latter cooling method (Eq. (20)) to be dominant, so we limit ourselves to using that.

To compute the effects due to neutrinos, we must know the temperature. However, in turn, we want to use the neutrino effects to find the temperature. We therefore first solve the energy Eq. (13) without adding neutrino effects. Then we use the resulting temperature to calculate the neutrino emissivity, which is then added to the total energy in Eq. (13), and this equation is solved to find the temperature as a function of distance travelled. The neutrinos are released in small successive bursts, mimicking a continuous emission. We again emphasize that, because of the neutrinos effects and the different energy equation used in Sect. 3.3, there is no assumption of energy equipartition applied in this section.

The temperature is shown in Fig. 7 as a function of distance travelled. In general, the release of neutrinos are seen as a small jump in the temperature curve. We note that cases 1 to 4 reach the light cylinder with reasonable temperatures $\left(T<10^{12} \mathrm{~K}\right)$ and densities $\left(\rho<10^{14} \mathrm{~g} / \mathrm{cm}^{3}\right)$. Cases 5 and 7 are ruled out, as they reach even more extreme conditions before reaching the light cylinder. For illustrative purposes in Fig. 8 we show the energy components for case 3 . Because of the neutrino effects, the radiation energy is now the dominant energy, even close to the light cylinder.

\section{Sites and formation mechanisms}

In this section we discuss sites that are best suited to account for the $\mathrm{CB}$ conditions at the source derived in our previous section. We also explore possible formation scenarios.

\section{1. "Standard" and hyperaccreting disks}

In Appendix A we summarize the properties of "standard" ( $\alpha$ disks and advection-dominated accretion flow disks) and hyperaccretion disks. It is clear that "standard" accretion disks are ruled out. Hyperaccreting disks, on the other hand, are candidates for CB sources. They have densities, temperatures, and magnetic fields that are comparable to what we found in the previous section. In fact when we consider CBs with hyperaccretion disk conditions at the source and perform a forward integration, the conditions at $D_{\text {trans }}$ (see Figs. B.1-B.3 in 


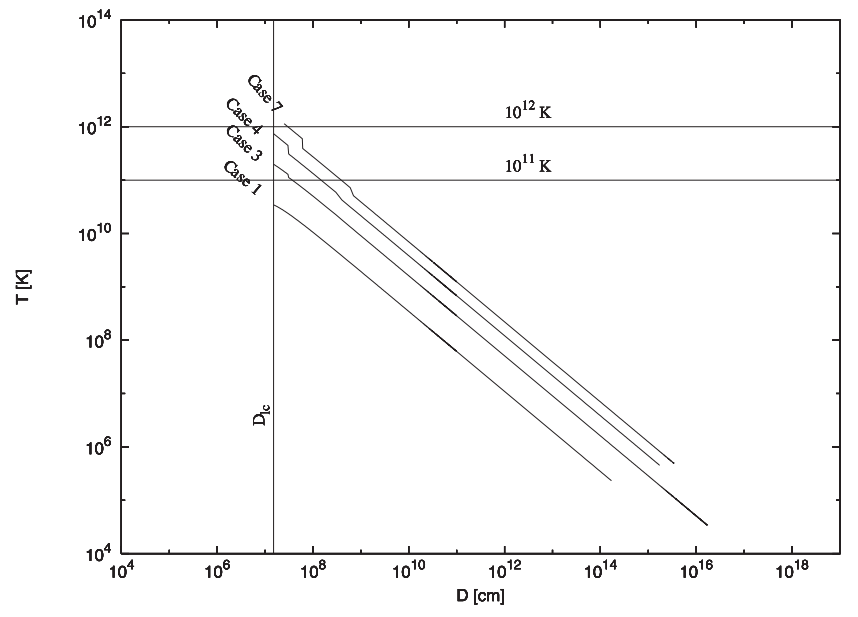

Fig. 7. The temperature assuming the same total energy as in Sect. 3.3 with neutrino and degeneracy effects. Case 7 goes back to $10^{12} \mathrm{~K}$ (at $D=2.5 \times 10^{12} \mathrm{~cm}$ ), whereas case 1,3 , and 4 goes to the light cylinder.

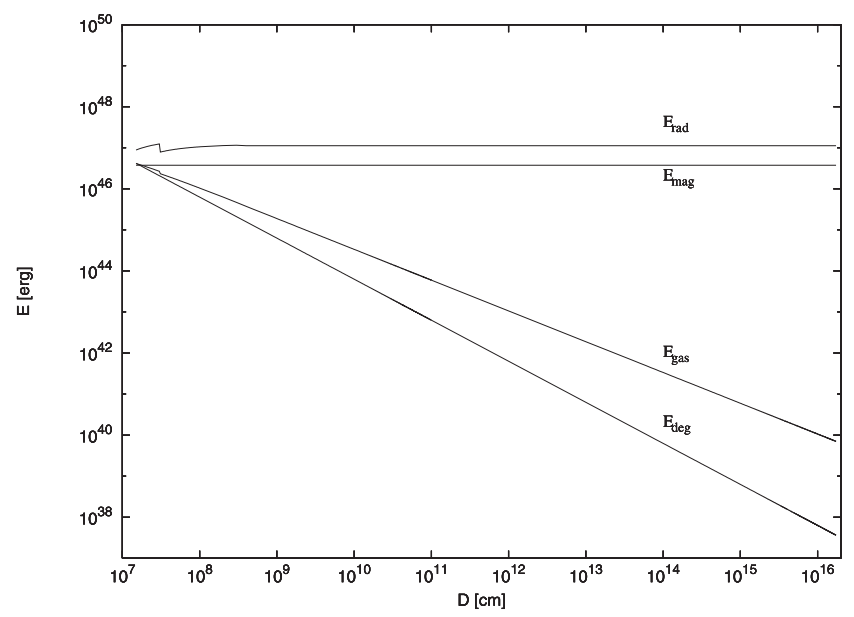

Fig. 8. The energy components for case 3, including degeneracy and neutrino effects, and assuming that case 3 originate at the light cylinder. The neutrino release is visible in the radiation energy and gas thermal energy at $D=3 \times 10^{7} \mathrm{~cm}$. Another non visible release occurs at $D=3 \times 10^{8} \mathrm{~cm}$.

Appendix B) turn out to be interestingly similar to those given by Dado et al. (2002). The next step then is to look for formation mechanisms within the hyperaccreting context.

The stability of hyperaccreting disks around black holes have been recently investigated by Di Matteo et al. (2002), who find that the "flows are gravitationally stable under almost all conditions of interest". Exceptions exist for strong accretion rates and in the outer part of the disk (see also Narayan et al. 2001). However as can be seen for Eqs. (A.8)-(A.11) in the appendix, these extreme cases favor lower densities and temperatures than those expected for CBs.

The magneto-rotational instability (MRI, Balbus \& Hawley 1991; and Hawley \& Balbus 1991) works only for low magnetic field strengths and cannot account for the strong magnetic fields required at the origin for CBs. Let us also mention the accretion-ejection instability (Tagger et al. 1999) as a possible formation mechanism. This instability works for intermediate magnetic field strengths and will transfer angular momentum to Alfvén waves toward the corona of the disk. At extreme magnetization the accretion-ejection instability is reminiscent of the interchange instability (Spruit et al. 1995), but it seems unlikely that these can lead to CB formation since most of the perturbations are carried by Alfvén waves.

It is thus not clear how a $\mathrm{CB}$ can form within a hyperaccretion disk. There is also the issue of accelerating the $\mathrm{CB}$ to $\Gamma>100$, which is also a major challenge. We will return to this in Sect. 5 after we discuss other possible formation sites.

\subsection{Neutron tori}

The thick, self-gravitating, neutron tori around $2-3 M_{\odot}$ black holes are known to be affected by a runaway instability on time scales below the evolutionary time scale of GRBs (Nishida \& Eriguchi 1996), so we exclude them as sources for CBs. Simulations of neutron star mergers have also shown that about $0.01 M_{\odot}$ of the thick disk of $0.2 M_{\odot}$ around a 1.5 to $3.1 M_{\odot}$ final central mass distribution becomes gravitationally unbound (Rosswog et al. 1999). However, in contrast to the hyperaccreting disk model, this unbound mass stays rather cold $\left(10^{8} \mathrm{~K}\right)$ and does not constitute a formation site for CBs.

\subsection{Accretion disk corona}

Another possibility is CB formation in the disk corona, for example, as a huge magnetic flare that ejects a large part of the accretion disk corona into a bullet of high velocity. A CB of such a size would have a density of about $2.4 \times 10^{5} \mathrm{~g} \mathrm{~cm}^{-3}$, which, for comparison is in the range of white dwarf densities. The maximum initial size of the $\mathrm{CB}$ we do not expect to exceed $R_{\mathrm{LC}} \simeq 10^{7} \mathrm{~cm}$. Comparing the $\mathrm{CB}$ asymptotic kinetic energy to the magnetic energy contained in a volume of that size provides an estimate for the mean magnetic field strength of about $10^{15} \mathrm{G}$. This corresponds to a magnetic flux of $\sim 10^{29}-10^{30} \mathrm{G} \mathrm{cm}^{2}$ and is unrealistically high for such coronae.

\subsection{Disk-jets and funnel-jets}

Figure 9 is an illustration of the type of jets that could emanate from the vicinity of a compact star. The disk-jet material is ejected from the accretion disk, while the funnel-jet is ejected from the innermost parts of the disk at the interface with the compact star.

Recent general relativistic magneto-hydrodynamic simulations by De Villiers et al. (2005) of a black hole and an initial torus seeded with a weak poloidal magnetic field show that a funnel jet with $\Gamma_{\mathrm{CB}} \gtrsim 50$ is formed. Instabilities do occur in funnel-jets, however the induced instabilities have much lower densities than the $\mathrm{CB}$ values found in the previous section. Funnel-jets can therefore be ruled out as a possible formation site for CBs.

A disk-jet becomes cylindrically collimated on a length scale on the order of 1-2 light cylinder distances (Fendt \& Memola 2001). Knot-generating instabilities reminiscent of 


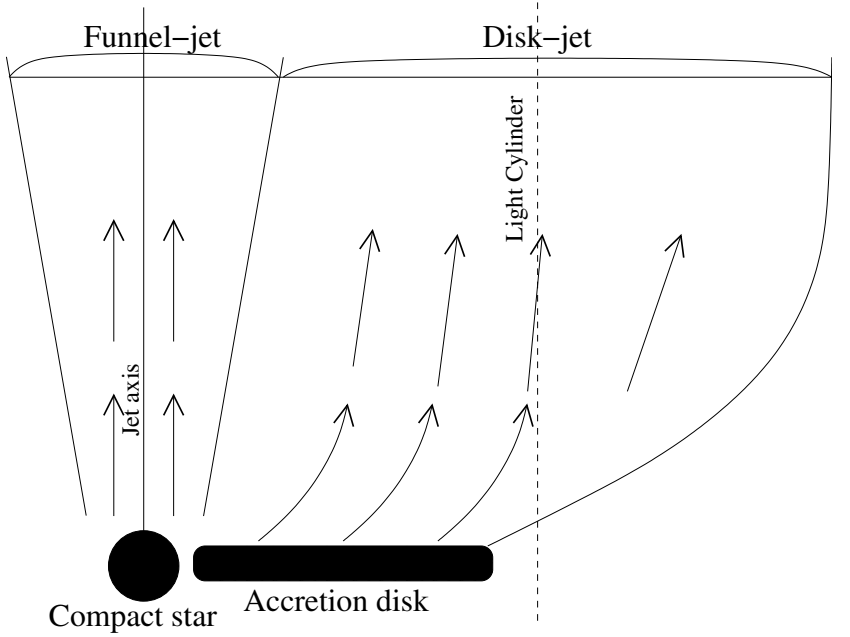

Fig. 9. Illustration of the funnel-jet and disk-jet. The funnel-jet is launched from a region close to the compact star. The disk-jet is launched from the accretion disk.

CBs are known to occur as jets collimate (Ouyed et al. 1997). This is a possible formation mechanism for CBs. What remains is to show how they can be accelerated to high $\Gamma$.

\section{Cannonball acceleration to ultra-relativistic velocity}

Having isolated, or more precisely favored, jets from hyperaccretion disks as plausible formation sites for $\mathrm{CBs}$, we now discuss those acceleration mechanisms with which $\mathrm{CBs}$ can reach Lorentz factors in the thousands.

Assuming that the $\mathrm{CB}$ is accelerated by converting the internal magnetic energy to kinetic energy, we can find an estimate for the magnetic field needed to explain such Lorentz factors. Using typical $\mathrm{CB}$ radii close to the source and parameters from Table 1, we find that the magnetic field must be on the order of $10^{18}-10^{19} \mathrm{G}$. This is unrealistically high. Either our approach is too simple or a different acceleration mechanism must be at work.

\subsection{MHD acceleration: $C B$ speed}

The ability of the magnetic field to accelerate particles to high Lorentz factors is given by the magnetization parameter (Michel 1969)

$\sigma=\frac{\Phi^{2} \Omega_{\mathrm{F}}^{2}}{4 \dot{M}_{\mathrm{jet}} c^{3}}$,

where $\Phi=B_{\mathrm{p}} r^{2}$ is the magnetic flux, $\Omega_{\mathrm{F}}=c / D_{\mathrm{lc}}$ the angular frequency of the magnetic field, and $\dot{M}_{\mathrm{jet}}=\pi \rho v_{\mathrm{p}} r^{2}$ the mass flow rate within the flux surface. For spherical outflow Michel (1969) found that the Lorentz factor at infinity scales as

$\Gamma_{\infty}=\sigma^{1 / 3}$.

Fendt \& Ouyed (2004) finds a modified Michel scaling in the case of a non-spherical magnetic field distribution. In this case they find a linear relation between $\sigma$ and $\Gamma_{\infty}$. If the field distribution is $\Phi(r ; \Psi) \sim r^{-0.1}$, they find that $\Gamma_{\infty}=10^{-1 / 3} \sigma$; and if $\Phi(r ; \Psi) \sim r^{-0.2}$, they find

$\Gamma_{\infty}=10^{-1 / 5} \sigma$,

in which case hyperaccreting disks with ejection rates on the order of $10^{-5} M_{\odot} / \mathrm{s}$ and magnetic field on the order of $10^{14} \mathrm{G}$ can lead to jets with a Lorentz factor $\Gamma_{\infty} \simeq 1875$.

\section{2. $M H D$ instability: $C B$ mass}

To a first order, instabilities related to Alfvén crossing time can develop on timescales

$t_{\text {ins }}=t_{\mathrm{A}}=\frac{2 R_{\text {jet }}}{v_{\mathrm{A}}}$,

where $R_{\text {jet }}$ is the radius of the disk-jet. For $1 R_{\mathrm{lc}}<R_{\text {jet }}<10 R_{\mathrm{lc}}$, we arrive at $t_{\text {ins }} \sim 1-10 \mathrm{~ms}$, which would imply the plausible formation of a blob of matter as massive as $M_{\text {ins }}=t_{\text {ins }} \dot{M}_{\text {jet }} \sim$ $10^{-8}-10^{-7} M_{\odot}$. This can be compared to the typical CB mass on the order $M_{\mathrm{CB}}=10^{-7} M_{\odot}$.

As shown above, first forming the $\mathrm{CB}$ in the disk and then accelerating it will require unrealistic magnetic fields on the order $10^{19} \mathrm{G}$. However, first accelerating the wind to the light cylinder and then forming the $\mathrm{CB}$ through an instability beyond the light cylinder requires much smaller magnetic field strength $\left(<10^{14} \mathrm{G}\right)$. This is a possible mechanism for forming and accelerating CBs.

\section{Conclusion}

Assuming that CBs move and expand with constant velocity, we have estimated the $\mathrm{CB}$ conditions as close as possible to their origin. Cannonballs require extremely high internal magnetic fields when they are formed with a field strength exceeding $\sim 10^{15} \mathrm{G}$. The temperature was found to be on the order of $10^{11}-10^{12} \mathrm{~K}$. The physical parameters of the CBs at the origin are indicative of hyperaccreting disks, within an order of magnitude estimates. However, if they are formed in the accretion disk we find it challenging to accelerate the CBs to the high Lorentz factors. The coronal origin is ruled out because of the unrealistically high coronal magnetic flux necessary to form the CBs. Our results instead hint at a jet origin for CBs. The radius $\left(<D_{\mathrm{lc}}\right)$ and mass flow $\left(10^{-5} M_{\odot} / \mathrm{s}\right)$ in a jet from a hyperaccreting disk can account for the CB mass and density. Furthermore, this outflow can be accelerated to $\Gamma \sim 2000$ by MHD processes (Fendt \& Ouyed 2004). Any instability in this outflow beyond the light cylinder could lead to CB formation. We thus suggest that CBs form as instabilities in ultrarelativistic jets emanating from the surface of hyperaccretion disks. The tight link between $\mathrm{SNe}$ and the $\mathrm{CB}$ model for GRB requires that all (or almost all) core-collapse $\mathrm{SNe}$ will produce CBs. Our work, within its limitations, implies that hyperaccretion disks must be a common occurrence in core collapse $\mathrm{SNe}$ to accommodate the $\mathrm{CB}$ model - a notion which remains to be confirmed.

Acknowledgements. The research of R.O. is supported by an operating grant from the Natural Science and Engineering Research Council 
of Canada (NSERC), as well as the Alberta Ingenuity Fund (AIF). J.S. thanks the Canadian Institute for Theoretical Astrophysics for its hospitality. C.F. thanks R.O. for the gracious hospitality at the University of Calgary where much of this work was completed.

\section{References}

Balbus, S. A., \& Hawley, J. F. 1991, ApJ, 376, 214

Dado, S., Dar, A., \& De Rújula, A. 2002, A\&A, 388, 1079

Dar, A., \& De Rújula, A. 2004, Phys. Rep., 405, 203

De Villiers, J. P., Staff, J., \& Ouyed, R. 2005 [arXiv:astro-ph/0502225]

Di Matteo, T., Perna, R., \& Narayan, R. 2002, ApJ, 579, 706

Fendt, Ch., \& Memola, E. 2001, A\&A, 365, 631

Fendt, Ch., \& Ouyed, R. 2004, ApJ, 608, 378

Hawley, J. F., \& Balbus, S. A. 1991, ApJ, 376, 223
Michel, F. C. 1969, ApJ, 158, 727

Narayan, R., \& Yi, I. 1995, ApJ, 452, 710

Narayan, R., Mahadevan, R., \& Quataert, E. 1998, in The Theory of Black Hole Accretion Discs, ed. M. A. Abramowicz, G. Bjornsson, \& J. E. Pringle (Cambridge: Cambridge Univ. Press), 148

Narayan, R., Piran, T., \& Kumar, P. 2001, ApJ, 557, 949

Nishida, S., \& Eriguchi, Y. 1996, ApJ, 461, 320

Ouyed, R., Pudritz, R. E., \& Stone, J. M. 1997, Nature, 385, 4090

Piran, T. 1999, Phys. Rep., 314, 757

Popham, R., Woosley, S., \& Fryer, Ch. 1999, ApJ, 518, 356

Rosswog, S., Liebendörfer, M., Thielemann, F.-K., et al. 1999, A\&A, 341,499

Shakura, N. I., \& Sunyaev, R. A. 1973, A\&A, 24, 337

Spruit, H. C., Stehle, R., \& Papaloizou, J. C. B. 1995, MNRAS, 275, 1223

Tagger, M., Pellat, R., \& Coroniti, F., 1992, ApJ, 393, 708 


\section{Online Material}


J. E. Staff et al.: Cannonball formation sites, Online Material p 2

\section{Appendix A: Accretion disks}

\section{A.1. "Standard" accretion disks}

A standard Shakura-Sunyaev disk (Shakura \& Sunyaev 1973) will have a density

$$
\begin{aligned}
\rho\left[\mathrm{g} \mathrm{cm}^{-3}\right]= & 7.2 \times 10^{-4}\left(\frac{\alpha_{\mathrm{v}}}{0.001}\right)^{-1}\left(\frac{\dot{M}}{M_{\mathrm{Edd}}}\right)^{-2} \\
& \times\left(\frac{r}{3 r_{\mathrm{S}}}\right)^{3 / 2} \times\left(\frac{M}{M_{\odot}}\right)^{-1}\left(1-\left(\frac{r}{3 r_{\mathrm{S}}}\right)^{-1 / 2}\right)^{-2},
\end{aligned}
$$

where $\alpha_{\mathrm{v}}$ is a viscosity parameter, $r_{S}$ is the Schwarzschild radius, and $M_{\mathrm{Edd}}$ is the Eddington mass. With this density, the radius of a $\mathrm{CB}$ with mass $M=10^{50}$ baryons becomes $3.8 \times 10^{9} \mathrm{~cm}$, assuming the default parameters. For the equipartition magnetic field, one gets

$$
B[\mathrm{G}]=10^{8}\left(\frac{M}{M_{\odot}}\right)^{-1 / 2}\left(\frac{r}{3 r_{\mathrm{S}}}\right)^{-3 / 4} .
$$

The temperature is

$$
T[\mathrm{~K}]=1.3 \times 10^{8}\left(\frac{\alpha_{\mathrm{v}}}{0.001}\right)^{-1 / 4}\left(\frac{M}{M_{\odot}}\right)^{-1 / 4}\left(\frac{r}{r_{\mathrm{S}}}\right)^{-3 / 4} .
$$

Advection Dominated Accretion Flow (ADAF) disks have density (Narayan \& Yi 1995):

$$
\begin{aligned}
\rho\left[\mathrm{g} \mathrm{cm}^{-3}\right]= & 6.5 \times 10^{-3}\left(\frac{\alpha_{\mathrm{v}}}{0.001}\right)^{-1} c_{1}^{-1} c_{3}^{-1 / 2} \\
& \times\left(\frac{\dot{M}}{M_{\text {Edd }}}\right)^{+1} \times\left(\frac{M}{M_{\odot}}\right)^{-1}\left(\frac{r}{3 r_{\mathrm{S}}}\right)^{-3 / 2},
\end{aligned}
$$

where $c_{1}$ and $c_{3}$ are defined in Eq. (2.1) in Narayan \& Yi (1995).

The corresponding magnetic field is

$$
\begin{aligned}
B[\mathrm{G}]= & 5.5 \times 10^{9}\left(\frac{\alpha_{\mathrm{v}}}{0.001}\right)^{-1 / 2} c_{1}^{-1 / 2} c_{3}^{1 / 4}(1-\beta)^{1 / 2} \\
& \times\left(\frac{\dot{M}}{M_{\mathrm{Edd}}}\right)^{1 / 2}\left(\frac{M}{M_{\odot}}\right)^{-1 / 2}\left(\frac{r}{3 r_{\mathrm{S}}}\right)^{-5 / 4},
\end{aligned}
$$

while the ion temperature of such disks are (Narayan et al. 1998)

$T_{\mathrm{i}}[\mathrm{K}]=2 \times 10^{12} \beta\left(\frac{r}{2 r_{\mathrm{s}}}\right)^{-1}$,

where $\beta$ is given by

$p_{\mathrm{m}}=\frac{B^{2}}{24 \pi}=(1-\beta) \rho c_{\mathrm{s}}^{2}$.

\section{A.2. Hyperaccreting disks}

Hyperaccreting disk (Popham et al. 1999) density is

$$
\begin{aligned}
\rho\left[\mathrm{g} \mathrm{cm}^{-3}\right]= & 1.3 \times 10^{12}\left(\frac{\alpha_{\mathrm{v}}}{1.0}\right)^{-1.3}\left(\frac{\dot{M}}{M_{\odot} s^{-1}}\right)^{+1} \\
& \times\left(\frac{M}{M_{\odot}}\right)^{-1.7}\left(\frac{r}{3 r_{\mathrm{S}}}\right)^{-2.55} ;
\end{aligned}
$$

their disk scale height is

$H[\mathrm{~cm}]=1.9 \times 10^{5}\left(\frac{\alpha_{\mathrm{v}}}{1.0}\right)^{0.1}\left(\frac{M}{M_{\odot}}\right)^{0.9}\left(\frac{r}{3 r_{\mathrm{S}}}\right)^{1.35}$,

while the temperature is

$T[\mathrm{~K}]=7.6 \times 10^{10}\left(\frac{\alpha_{\mathrm{v}}}{1.0}\right)^{0.2}\left(\frac{M}{M_{\odot}}\right)^{-0.2}\left(\frac{r}{3 r_{\mathrm{S}}}\right)^{-0.3}$.

The corresponding equipartition magnetic field is on the order of

$B[\mathrm{G}] \sim 10^{14}-10^{15}$.

\section{Appendix B: Forward integration}

For completeness and as a self-consistency check, here we consider CBs with hyperaccreting disk conditions at the origin and perform a forward integration until the $\mathrm{CBs}$ reach the distances where they become transparent.

Assuming that the $\mathrm{CB}$ radius evolves as before, $R=$ $c /\left(\Gamma_{\mathrm{CB}} \sqrt{3}\right)$, then the density at a distance corresponding to the surface of the hyperaccreting disk $\left(D=10^{5} \mathrm{~cm}\right)$ will be too high. We will thus make a slight adjustment by rewriting the radius as $R=D /\left(\Gamma_{\mathrm{CB}} \sqrt{3}\right)+x$, where $x$ is a number that ensures that the density at the origin does not exceed $\rho=10^{12} \mathrm{~g} / \mathrm{cm}^{3}$. Therefore, $x$ is found by solving the following equation:

$10^{12} \mathrm{~g} / \mathrm{cm}^{3}=\frac{M_{\mathrm{CB}}}{\frac{4}{3} \pi\left(\frac{10^{5} \mathrm{~cm}}{\Gamma_{\mathrm{CB}} \sqrt{3}}+x\right)^{3}}$,

which implies:

$x[\mathrm{~cm}]=\frac{3 M_{\mathrm{CB}}^{1 / 3}(6 / \pi)^{1 / 3} \Gamma_{\mathrm{CB}}-\sqrt{12} \times 10^{9}}{60000 \Gamma_{\mathrm{CB}}}$.

This also ensures the correct expansion velocity $v_{\exp }=c / \sqrt{3}$. Table B. 1 shows the corresponding parameter values for $x$ and $\epsilon_{\mathrm{m}}$. We should also note that in this case $\epsilon_{\mathrm{m}}$ will be chosen so as to insure energy equipartition at the disk surface. The temperature thus found is used to calculate the neutrino emissivity, which is then added to the total energy in Eq. (13) to find the new temperature. As before, the neutrinos are released in small successive bursts that mimic a continuous release of neutrinos.

Figures B.1-B.3 show the temperature, magnetic field and density as a function of distance. All cases starts with $T \sim 2 \times$ $10^{11} \mathrm{~K}, \rho=10^{12} \mathrm{~g} / \mathrm{cm}^{3}$, and $B=6 \times 10^{16} \mathrm{G}$ at $D=10^{5} \mathrm{~cm}$. The neutrino effects can be seen as small jumps in the temperature curves, but in general the neutrinos do not change the overall picture a lot. The neutrino contribution were on the same order as or smaller than the total energy, and as discussed before, the temperature is not very sensitive to changes in the total energy.

The temperature at $D_{\text {trans }}$ is the same order as for the backward integration $\left(T=10^{4} \mathrm{~K}\right.$ to $T=10^{5} \mathrm{~K}$ ) and also close to the value given by Dado et al. (2002) of $T_{\text {trans }} \simeq 4 \mathrm{eV}$. The difference between the backward and forward integration at large distances is due to the different $\epsilon_{\mathrm{m}}$ parameters. For large distances, the $x$-parameter does not play any role.

To summarize, the results of the forward integration indicate that CBs formed within hyperaccretion disks could theoretically provide the necessary conditions at $D_{\text {trans }}$ to account for GRB features as claimed in Dado et al. (2002). 
J. E. Staff et al.: Cannonball formation sites, Online Material p 3

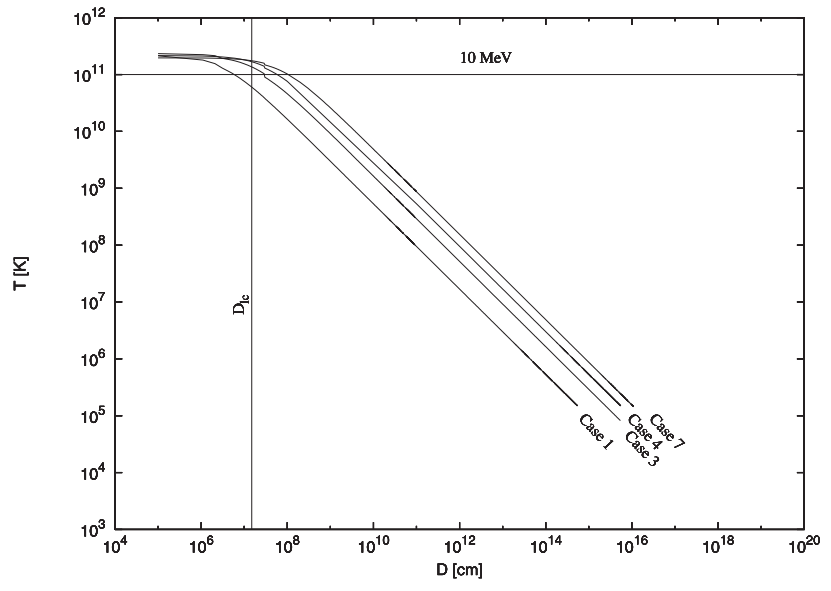

Fig. B.1. Temperature vs. distance from the origin for the CB, starting with hyperaccreting disk conditions. The forward integration is stopped when the CB becomes transparent to its enclosed radiation. The initial temperature for all cases is about $2.7 \times 10^{11} \mathrm{~K}$.

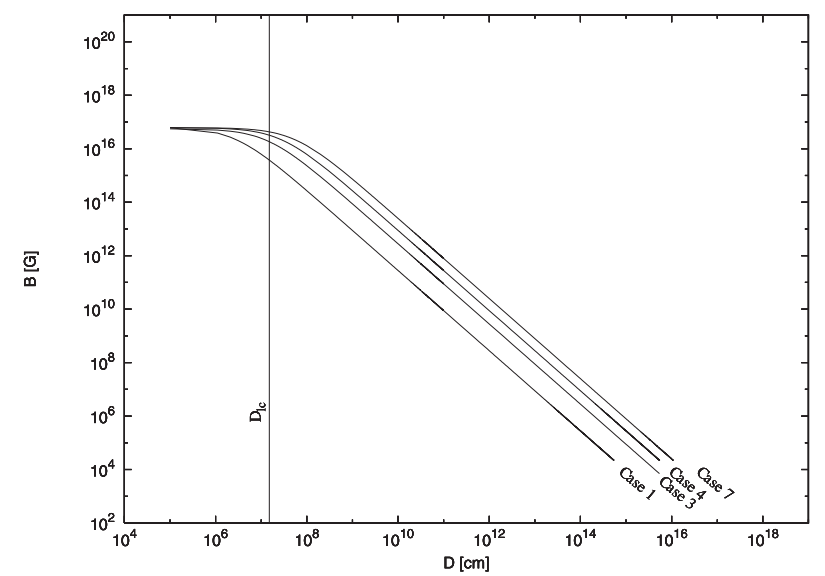

Fig. B.2. Magnetic field vs distance from the origin for the CB, starting with hyperaccreting disk conditions. The forward integration is stopped when the CB becomes transparent to its enclosed radiation. The initial magnetic field for all cases is about $5 \times 10^{16} \mathrm{G}$.

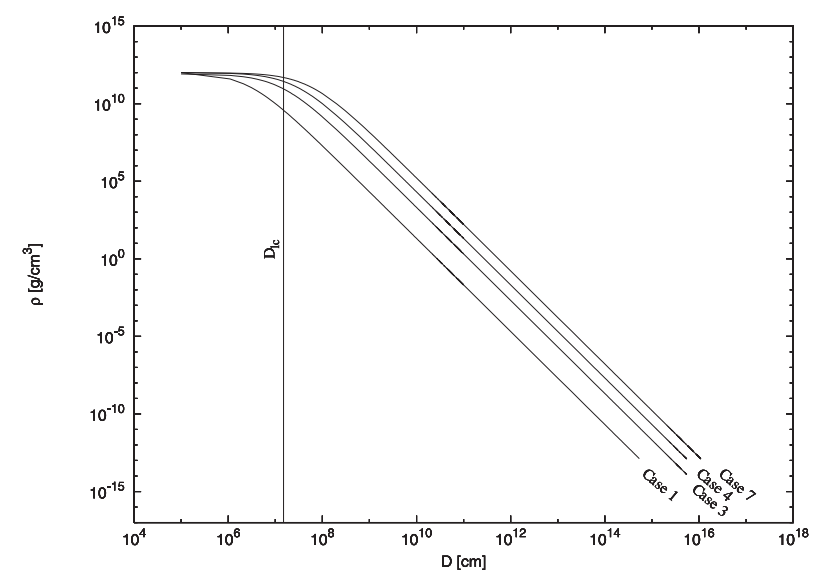

Fig. B.3. Density vs distance from the origin for the CB, starting with hyperaccreting disk conditions. The forward integration is stopped when the $\mathrm{CB}$ becomes transparent to its enclosed radiation. The initial density for all cases is about $1 \times 10^{12} \mathrm{~g} / \mathrm{cm}^{3}$.
Table B.1. The parameter $x$ used in the relation between $R_{\mathrm{CB}}$ and $D$, and $\epsilon_{\mathrm{m}}$ for the different $\mathrm{CB}$ cases when starting with disk conditions and integrating forward.

\begin{tabular}{lll}
\hline \hline Case & $x[\mathrm{~cm}]$ & $\epsilon_{\mathrm{m}}$ \\
\hline 1 & 15279.2 & 0.045 \\
2 & 33584.5 & 0.045 \\
3 & 73022.3 & 0.045 \\
4 & 15798.9 & 0.045 \\
5 & 34104.2 & 0.045 \\
7 & 15827.7 & 0.045 \\
\hline
\end{tabular}

